Abstract

\title{
Prevalence of Gene Variants Associated with Poor Absorption or Negative Interactions with Key Anti- Inflammatory Nutrients in a New Zealand Population ${ }^{\dagger}$
}

\author{
Bobbi Brennan Laing 1,*, Nishi Karunasinghe ${ }^{2}$ and Lynnette Robyn Ferguson ${ }^{2}$ \\ 1 School of Nursing, University of Auckland, Auckland 1023, New Zealand \\ 2 Auckland Cancer Society Research Centre, University of Auckland, Auckland 1023, New Zealand; \\ n.karunasinghe@auckland.ac.nz (K.N.); 1.ferguson@auckland.ac.nz (F.L.R.) \\ * correspondence: b.laing@auckland.ac.nz \\ + Presented at the 2018 Nutrition Society of New Zealand Annual Conference, Auckland, New Zealand, \\ 28-30 November 2018.
}

Published: 11 March 2019

Background: New Zealand (NZ) has high rates of Crohn's disease (CD) at 26/105. Having gene variants associated with low levels of betacarotene, vitamin D and Omega-3 polyunsaturated fatty acids can impede the immune response. Not known was the prevalence of these variants in the $C D$ population in NZ.

Methods: We determined the prevalence of these gene variants in NZ adults in two matched groups, one with CD $(n=416)$ and a control group of healthy adults $(n=649)$ selected from adult subjects in the 'Genes and Diet in Inflammatory Bowel Disease Study' of Nutrigenomics NZ. The selected SNPs included those associated with genes with betacarotene absorption BCMO1Betacarotene 15,15'-monooxygenase-1, (rs12934922, rs7501331); vitamin D concentrations in the genes GC-Group-specific component (rs2282679, rs4588, rs1155563), the Cytochrome P450 family: CYP24A1-(rs1699913), CYP2R1 (rs10741657), and DHCR7/NADSYN1 7-dehydrocholesterol reductase (rs3829251,rs12785878); with fatty acid desaturases genes which influence omega-three and-six fatty acid metabolism: FADS1, FADS2 (rs174556, rs174570, rs2072114, rs174583 \& rs174589); with the Peroxisome proliferator-activated- receptor genes relating to: cholesterol levels PPARA (rs4253728); and with CD activity. PPARG (rs1801282); X-ray repair cross-complementing protein 1, XRCC1 (rs25487) associated with colorectal adenoma, and SCD- Stearoyl-coA desaturase (rs 2060792) with inflammation. These genotypes were assessed using custom SNP Sequenom MassARRAY analyses.

Results: The three variants: TT in rs12934922, (BCM01); GG in rs10741657, (CYP2R1) and TT in rs174583 (FADS2) had a representation of more than $16 \%$. The- frequencies of these SNPs known to associate with low betacarotene absorption and vitamin $\mathrm{D}$ concentration and negative fatty acid interactions respectively, were 18, 39 and $16 \%$ in both the healthy as well as the CD groups in these cohorts.. These frequencies are similar to other reported healthy European groups of 24, 38 and 13\%.

Conclusion: Around $16 \%-39 \%$ of the NZ population maybe deprived of these anti-inflammatory nutrient requirements due to these variant genotypes.

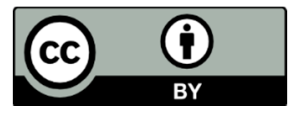

(C) 2019 by the authors. Licensee MDPI, Basel, Switzerland. This article is an open access article distributed under the terms and conditions of the Creative Commons Attribution (CC BY) license (http://creativecommons.org/licenses/by/4.0/). 